\title{
Transferência tecnológica da vacina de influenza no acordo entre 0 Instituto Butantan e a Sanofis/Pasteur
}

Technological transfer of influenza vaccine in the agreement between the Butantan Institute and Sanofis/Pasteur

1.

Professor adjunto de Medicina Social FCM da Santa Casa de São Paulo e Pesquisador do Centro de Memória do Instituto Butantan. nelson.ibanez@butantan.gov.br

2.

Pesquisadora Cientifica do Centro de Memória do Instituto Butantan. olga.alves@butantan.gov.br

3.

Doutor em Educação pela Universidade Estadual de Campinas, licenciatura plena em História pela Universidade do Grande ABC e Pedagogia pela Universidade Federal de São Paulo

4.

Diretoria do Centro de Memória do Instituto Butantan, pesquisadora científica do Instituto Butantan, mestrado em Arqueologia pelo Museu de Arqueologia e Etnologia da de São Paulo, doutorado em História Social pela Universidade de São Paulo, pós-doutoranda da Faculdade de Saúde Pública da Universidade de São Paulo.

\author{
Nelson Ibañez ${ }^{1}$ \\ Olga Sofia Fabergé Alves ${ }^{2}$ \\ Carlos Eduardo Dias ${ }^{3}$ \\ Suzana Fernandes ${ }^{4}$
}

\section{Resumo}

0 presente artigo objetiva, passados mais de cem anos da pandemia de gripe espanhola (1918-1919), analisar o processo de transferência tecnológica da vacina de influenza da Sanofis Pasteur para o Instituto Butantan. A metodologia da pesquisa foi a de "estudo de caso" (YIN, 1994), respondendo às questões do "porquê" e do "como" ocorreu essa transferência. Analisa-se os contextos nacional e internacional no período, valendo-se de uma revisão bibliográfica do tema e realiza-se ainda análise documental e entrevistas semiestruturadas com atores-chave. As questões relativas ao "porquê" da transferência foram vistas de forma a identificar os aspectos envolvidos com a tomada de decisão, a relevância e a oportunidade de criação de conhecimento tecnológico por parte do setor público na produção em larga escala da vacina de influenza. As questões relativas ao "como", ou seja, ao processo em si da transferência, objetivaram identificar e analisar: os instrumentos formais (contrato de transferência de tecnologia), as etapas de implementação, fatores intervenientes, relações entre parceira (Sanofis) e Instituto, entraves institucionais, impactos econômicos e por fim discutir os resultados e as possibilidades de 
inovação criadas por esse processo. Os resultados alcançados pela transferência trazem à tona uma série de situações positivas e possibilidades de ganhos com a produção local e seu potencial inovador, o que na área social se dá pelo custo-benefício do ponto de vista da economia devido ao impacto nas internações e medicamentos necessários para o tratamento das pessoas contaminadas pela gripe.

\title{
Palavras-chave
}

vacina de influenza, transferência tecnológica, Instituto Butantan.

\begin{abstract}
This article aims, in the centenary of the Spanish Influenza Pandemic that occurred in 1918, to analyze the recent process of influenza vaccine production through the transfer in the agreement between the Butantan Institute and Sanofis/Pasteur. The methodology used in the research was that of a "case study" (YIN, 1994), answering the questions of "why" and "how" this transfer occurred. The national and international context was analyzed in the referred period, using a literature review on the subject. A documental analysis and semi-structured interview with key actors are also carried out. The questions related to the "why" of the transfer were seen in order to identify the aspects involved in the decision making, the relevance and the opportunity for the creation of technological knowledge by the public sector in the production of influenza vaccine. The questions related to the "how", that is, the transfer process itself, aimed to identify and analyze: the formal instruments (technology transfer contract), the stages of implementation and transfer and intervening factors, relations between partner (Sanofi) an and Institute; institutional barriers; economic impacts and, finally, discuss the results and possibilities of innovation created by this process. The results achieved by the transfer process bring to light a series of positive situations and possibilities for gains with local production and its innovative potential, which in the social area is given by the cost-benefit from the point of view of the economy due to the impact on hospitalizations and medication needed for the treatment of people contaminated by influenza.
\end{abstract}


A causa etiológica da gripe, a familia ortomixovirus, foi descoberta pela primeira vez em porcos por Richard Shope em 1931. A esta descoberta, seguiu-se em 1933 o isolamento do virus humano por parte de um grupo liderado por Patrick Laidlaw no Reino Unido.

\section{Keywords}

Influenza vaccine. Technology transfer. Butantan Institute.

\section{Introdução}

0 presente artigo objetiva, no centenário da pandemia de gripe espanhola ocorrida em 1918 e 1919, analisar o processo recente de produção da vacina de influenza mediante 0 acordo de transferência de tecnologia da Sanofi Pasteur para o Instituto Butantan (IBu). Esse processo visou a formulação, produção, envase, controle de qualidade da vacina de influenza, no período de 1999 a 2011, transformando o Butantan no primeiro produtor da vacina no hemisfério sul.

Um breve histórico desse evento situa de certa maneira como os serviços responsáveis por medidas de combate à gripe espanhola, incluindo o IBu, agiram no período evidenciando um desconhecimento científico do agente etiológico'. Em 1918, o estado epidêmico em São Paulo foi decretado por Arthur Neiva, Diretor do Serviço Sanitário. As medidas profiláticas tomadas para debelar o surto iam desde a proibição de aglomerações públicas até o fechamento de escolas e faculdades no município de São Paulo. Foram instalados nesses locais diversos hospitais provisórios para a assistência à população enferma, conforme nos informa Altino Arantes: "(...) com este [Arthur Neiva] combinei também o fechamento dos Grupos Escolares da Capital, podendo os respectivos edifícios ser convertidos em enfermarias para os indigentes atacados de grippe". (ARANTES, 1918).

A mobilização de todos os hospitais públicos, agentes do Serviço Sanitário de São Paulo e da Faculdade de Medicina na figura de seu Diretor Arnaldo Vieira de Carvalho, que colocou o corpo docente e discente da instituição a serviço dos 40 hospitais provisórios, conduziram as primeiras medidas de combate à gripe, contando com algumas instituições privadas (Companhia Antarctica, a Companhia Nacional de Juta, a Cristaleira Itália, a "Comissão Estado-Fanfulla"), filantrópicas (Cruz Vermelha Brasileira, Liga Nacionalista) e religiosas (Igreja Católica, Associação dos Pastores Evangélicos), se reuniram em solidariedade aos enfermos, passando a prestar serviços de tratamentos aos mesmos (SEIDL, 1919). 
2.

INSTITUTO BUTANTAN. Relatório de gestão: 1918. Acervo do Centro de Memória do Instituto Butantan.
0 Desinfectório Central passou a trabalhar dia e noite, para atender os pedidos de desinfecção nas casas onde ocorriam casos de gripe, principalmente os de gripe pneumônica. 0 Desinfectório também foi responsável pela remoção de doentes para os hospitais e de cadáveres para sepultamento (MEYER \& TEIXEIRA, 1920).

As medidas profiláticas adotadas na capital paulista durante o surto de gripe ocasionaram uma série de mudanças no dia a dia da população, estabelecendo uma espécie de "cotidiano epidêmico" no qual as pessoas procuravam seguir as prescrições médicas em um processo de reeducação. Mesmo assim, em vários momentos, as relações sociais e culturais resistiram ao flagelo desestruturador da epidemia de gripe e ao poder médico (BERTUCCI-MARTINS, 2002).

0 isolamento e a quarentena, medidas profiláticas mais adotadas pelos serviços oficiais em fases de epidemias, foram intensamente utilizados durante a fase mais aguda da pandemia gripal. Mas essa medida, geralmente polêmica, às vezes revelava a situação de desamparo vivida pela população.

A sugestão de alguns medicamentos no combate à doença também foi utilizada no combate à gripe, apesar do desconhecimento de seu agente causador e da não especificidade destes. A procura e o consumo foram intensos, causando desabastecimento nas farmácias públicas e privadas.

0 Instituto Butantan, subordinado ao Serviço Sanitário do Estado e responsável pela produção de soros e vacinas, pesquisa e difusão científica, dirigido à época por Vital Brazil, desempenhou seu papel compondo a rede de serviços que atendiam casos da gripe em sua enfermaria. Também realizou estudos e ensaios para o tratamento da gripe e ofertou produtos fabricados em suas dependências e comercializados pela "Casa Armbrust", em contrato firmado pelo Instituto (IBAÑEZ et al., 2006).

Um documento que registra sua atuação é o relatório de gestão de $1918^{2}$ assinado pelo Diretor Vital Brazil, que cita a atuação de um dos seus pesquisadores relacionada d̀ influenza: 
0 Dr. Afrânio do Amaral, com muita inteligência e atividade ocupou-se com observações clínicas e terapêuticas, colhidas principalmente na enfermaria de Butantan... Escreveu a respeito uma substanciosa e documentada memória que foi apresentada ḋ $2^{\circ}$ conferência de Higiene, além aspecto ocupou-se do ensaio de outros produtos do instituto, tais como o "Soro Normal" no tratamento da gripe, do soro antipneumocócica no tratamento da pneumonia gripal. (BUTANTAN, 1918, .5)

Verifica-se que, apesar do desconhecido agente causador da "gripe", os pesquisadores utilizavam produtos como o soro antipneumocócico e o "soro normal" produzido em cavalos. Este foi testado pelo Dr. Oscar Freire, auxiliado pelo Dr. Afrânio do Amaral, inicialmente na Santa Casa de Misericórdia e, posteriormente, em uma enfermaria na Faculdade de Farmácia, que ficou à disposição do Instituto, com resultados considerados satisfatórios (MEYER \& TEIXEIRA, 1920).

0 Instituto fez exames nos doentes recolhidos ao Hospital de Isolamento, encontrando, ao lado dos "bacillus influenzae de Pfeiffer", germens como o "pneumoccocus" e o "micrococcus catarrhalis", resultado encontrado em doentes de outras procedências. Como o mal se dava principalmente nos pulmões, o Butantan decidiu investir numa vacina polivalente com os microrganismos encontrados. Para isso foram incumbidos os médicos João Florêncio Gomes e Arlindo Raymundo de Assis, que desenvolveram uma vacina testada nos médicos e outros funcionários do Instituto, além de clínicas e na Força Pública (MEYER \& TEIXEIRA, 1920).

Outra forma da participação do Instituto na epidemia foi, como dissemos anteriormente, pelo fornecimento de produtos fabricados no instituto e comercializados pela "Casa Armbrust". Isto foi ilustrado pelo jornal 0 Estado de S. Paulo em 26 de outubro de 1918, que mostra ainda outros produtos indicados para a gripe pelos médicos de outros produtores além do Butantan. 
Imagem 1.

Recorte do jornal 0 Estado de

S. Paulo, 27 de outubro de 1918

Imagem 2.

Recorte do jornal 0 Estado de $S$.

Paulo, 27 de outubro de 1918, p.3

Imagem 3 e 4.

Recortes do jornal 0 Estado de S. Paulo, 27 de outubro de 1918, p.3
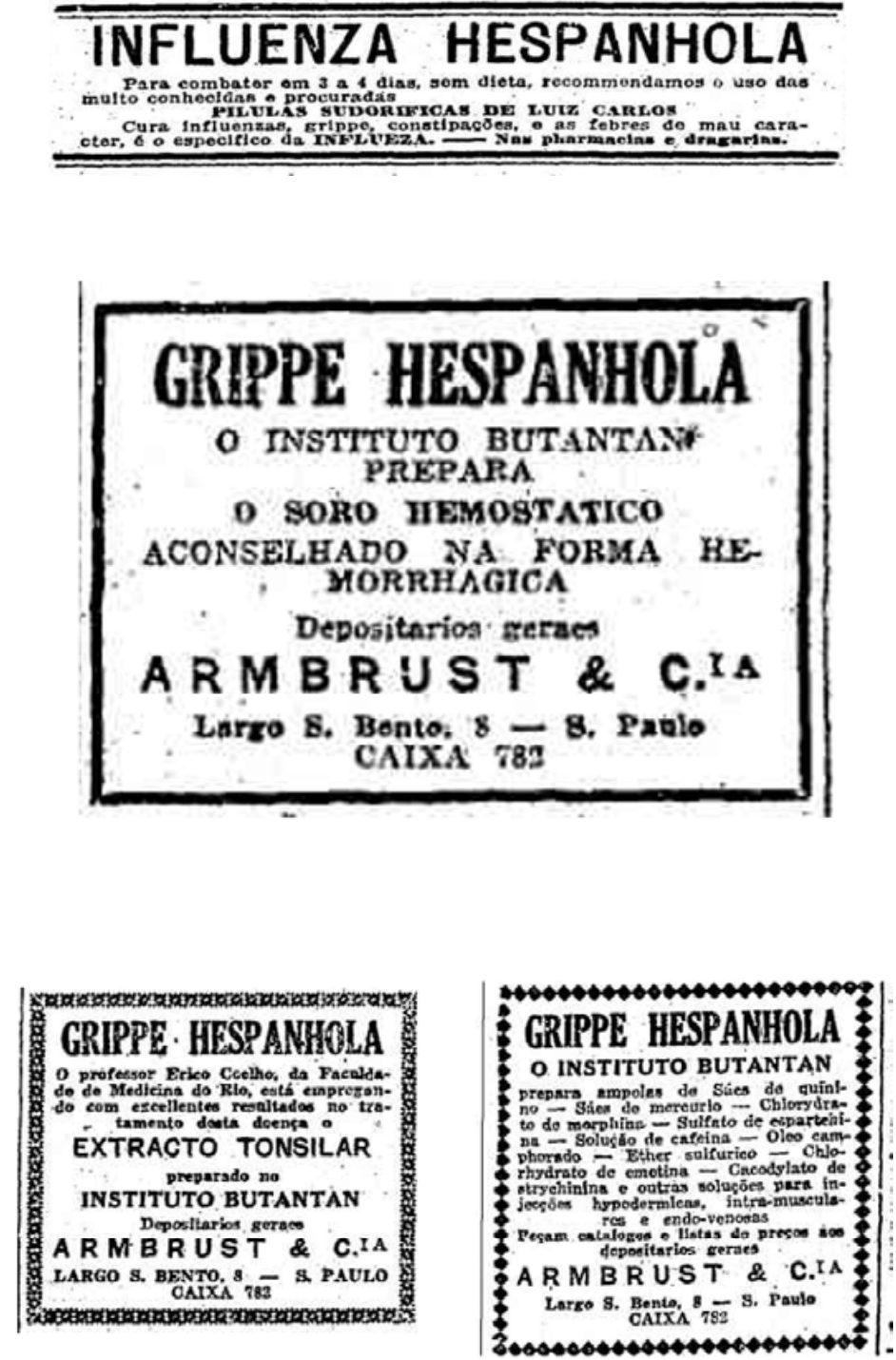

Nos anos seguintes, com o encerramento do contrato com a Casa Armbrust (ACOSTA, 2015), que resultou em uma série de debates sobre a distribuição de produtos e a interrelação entre o público e o privado, o Instituto Butantan passa por profundas transformações, cujo enfoque na pesquisa, está relacionado à criação de novas áreas dedicadas a patologias humanas que cresceram com a vinda de pesquisadores de outras instituições nacionais e internacionais. Entre a segunda metade de 1920 e a 
3.

Em 1944, foi desenvolvida uma vacina contra a gripe com vírus mortos, com base na investigação do australiano Frank Burnet, que demonstrou que o vírus perdia a virulência quando era cultivado em ovos de galinha. década de 1930, novos laboratórios experimentais foram inaugurados possibilitando algumas pesquisas pioneiras nas áreas de físico-química, genética e endocrinologia, por exemplo.

No entanto, mesmo com todos os investimentos em infraestrutura, foi somente na década seguinte, durante a direção de Eduardo Vaz, que a produção, vista então como atividade primordial do Instituto Butantan, retorna com a clara intenção de atender exclusivamente às demandas de saúde pública

No relatório de gestão de $1948^{3}$ é possivel verificar que o Instituto Butantan já havia preparado uma vacina experimental contra a influenza. Seu registro, redigido pelo então Diretor Eduardo Vaz, na seção de vírus, afirma:

"Vacina contra a gripe - Têm sido preparadas há mais de um ano pequenas partidas, e submetidas a prova de controle, não tendo sido até agora expedida nenhuma, pelo fato de ainda não satisfazerem as condições exigidas. Além disto a produção em escala que atenda às necessidades da Saúde Pública exige que se façam instalações maiores, e biotérios isolados dos demais, em que se trabalha com outros vírus". (INSTITUTO BUTANTAN, 1948)

As recomendações do relatório só seriam completamente implementadas anos depois, quando o Instituto Butantan inicia um processo de produção dessa vacina em larga escala, em acordo firmado com a empresa Sanofi Pasteur.

Devido ao risco de novas pandemias, o monitoramento do vírus influenza é realizado anualmente desde 1947, quando foi criada a Rede de Vigilância Epidemiológica pela OMS.

A pesquisadora aposentada do Instituto Butantan Dalva Mancini relata que a técnica de uso de ovo embrionado é a mesma desde o início da produção da vacina no Butantan, no final dos anos 1940, só que o vírus era de outro tipo: "Eu mesma tive oportunidade de ver no Instituto Butantan um lote, assim, já as ampolas de vacina preparada com a identificação: Influenza A H2N2. Então o Brasil já tinha competência para produzir na escala que pudesse, mas produzir essa vacina contra a Influenza AH2N2" (MANCINI, 2014). 
Entrevistas realizadas no período de 2012 a 2013 com diretores e pesquisadores do Instituto e da Fundação Butantan: Otávio Mercadante, Isaías Raw, Hernan Chaimovich, Cosue Miyaki,

Maurício Meros, Sally Prado.

5.

Edital Pesquisa para o SUS/

FAPESP/ Gestão Compartilhada em

Saúde PPSUS -SP, 2009
Mancini, que foi diretora do Laboratório de Virologia do Instituto Butantan, relata que trabalhou no desenvolvimento de vacina de influenza desde o início da década de 1990, a partir de protocolo adaptado da Organização Mundial de Saúde, optando pelo processo de ultra centrifugação dos vírus coletados no líquido alantóico dos ovos embrionados. Esse modelo de produção foi implantado por ela após estágio na empresa farmacêutica Boehringer na Alemanha. No Butantan, se fazia a vacina de modo artesanal, com pouco rendimento e destinada ao uso de um grupo limitado da população.

Em 1992 a produção do Butantan era de no mínimo 5 mil doses, dependendo da encomenda da Secretaria de Estado da Saúde, uma vez que a vacina não estava no PNI, e era distribuida para hospitais públicos para uso em grupos de risco, após avaliação pelos médicos no Hospital das Clínicas.

Desde 1977, a recomendação da Rede de Vigilância é que a composição da vacina contra gripe deve ser trivalente: duas cepas do tipo $A$, respectivamente dos subtipos H1N1 e H3N2, e uma do tipo B.

A composição das vacinas sazonais é atualizada anualmente com cepas coletadas pela Rede de Vigilância Global de Influenza da OMS. Desde 1999, a recomendação é que haja duas composições vacinais - uma para o Hemisfério Norte, e outra para o Sul. Nesse mesmo ano, o Ministério da Saúde decidiu incluir no programa nacional de imunização a vacinação contra influenza para indivíduos com mais de 65 anos de idade.

\section{Contexto, histórico e abordagem metodológica}

A análise do processo, objeto deste artigo, apoia-se num "estudo de caso" (YIN, 1994), visando responder às questões do "porquê" e do "como" foi feita essa transferência específica. A análise dos contextos nacional e internacional no período referido foi realizada a partir de uma revisão bibliográfica sobre o tema, além de análise documental e entrevistas semiestruturadas com atores-chave 4 . 0 presente artigo apoiou-se num estudo realizado pelo Laboratório de História da Ciência do IBu, que fez parte de projeto financiado pela FAPESP 5 , liderado pela Faculdade de Medicina da USP e denominado "Saúde, 
Publicação da Hucitec Editora São

Paulo, 2012
Desenvolvimento, Inovação Tecnológica e Cooperação Regional" (VIANA et al, 2012). ${ }^{6}$

0 Instituto Butantan é uma instituição pública de pesquisa, e de produção de vacinas e soros que tem como comprador oficial e único o Ministério da Saúde, o que se dá por conta das políticas universais de imunização e da garantia de acesso a insumos estratégicos desde meados da década de 1970. 0 desenvolvimento tecnológico de autossuficiência por parte do Brasil nas últimas décadas foi garantido pela presença de laboratórios e instituições públicas voltadas para a produção desses insumos.

Duas das estratégias traçadas pelo Ministério da Saúde nas décadas de 1970 e 1980 foram fundamentais nesse sentido: o estabelecimento do Plano Nacional de Imunização (PNI), em 1973, e o Programa de Autossuficiência Nacional (PASNI), em 1985. A criação do Sistema Único de Saúde (SUS) em 1988 ampliou consideravelmente a expansão dessas estratégias de incremento da produção nacional de imunobiológicos.

0 PNI foi estabelecido graças ao sucesso do programa de erradicação da varíola no Brasil. Já a emergência do PASNI, teve relação direta com a crise de suprimentos de imunobiológicos provocada pelo fechamento da Sintex do Brasil, as restrições às importações devido aos problemas na balança de pagamentos brasileira e a insuficiente produção local, já que diversos laboratórios públicos apresentavam condições inadequadas de funcionamento. Segundo Gadelha "a ideia básica do Ministério da Saúde era estabelecer uma ação coordenada entre os produtores nacionais, estimulando os investimentos e a melhoria de qualidade da produção local, de sorte a atingir a autossuficiência nos produtos vinculados aos programas nacionais." (GADELHA, 1996, p. 111)

As ideias centrais desenvolvidas pelos cientistas e estudiosos do tema foram assim expressas no que tange à agenda de desenvolvimento dos laboratórios públicos: a criação de competência para produção de vacinas, estimulando a pesquisa básica e aplicada; tratamento diferenciado para os institutos públicos vistos não somente como produtores, mas como centros de pesquisa; necessidade da presença do Estado nesta área, não apenas para se obter um preço compativel com os recursos disponiveis, mas porque a empresa privada nacional ou transnacional, 
mesmo com incentivos, ainda não investia em pesquisa no Brasil; e a existência de laboratórios públicos com capacidade tecnológica avançada para o desenvolvimento e a produção das chamadas vacinas 'órfãs', que não possuem importância comercial (GADELHA, 1996, p.125 e 127). Além disso, mostrou ser estratégico para o país à luz do PASNI, em função do que havia ocorrido com o fechamento da Sintex.

A questão da transferência tecnológica foi colocada no contexto de autossuficiência como uma das estratégias-chave, pois consiste em um conhecimento científico com valor agregado. Por este motivo a transferência de tecnologia não pode ser vista apenas como uma operação de compra e venda, pois compreende uma transação com operações explícitas e implícitas.

No caso especifico de uma transferência entre uma empresa privada com fins lucrativos é uma instituição produtora pública, esse processo pode apresentar, segundo Paulo Lee Ho e colaboradores (2011), problemas como: a relutância das empresas em criar concorrentes com conhecimentos e tecnologia mais atualizados e avançados; dependência permanente por materiais essenciais pertencentes às empresas; imposição de preço mínimo pelo qual a vacina possa ser vendida e, por fim, como a transferência é gradual, a exigência de continuar comprando a granel do fornecedor da tecnologia por um período longo.

Melo comenta que essa questão da transferência não pode ser restrita à alegação de que as empresas multinacionais não transferem tecnologia:

A globalização da economia, de certa forma, abriu perspectivas importantes para investimentos internacionais fora do espaço geográfico das suas matrizes. A questão é saber se seremos hábeis para atrair centros de pesquisa e unidades produtoras para fechar a articulação pesquisa/ produção. Nota-se um certo esgotamento em países europeus, como, por exemplo, a Inglaterra e os países nórdicos. (MELO, 1996, p.119-120).

A existência do PASNI e a obrigação do Ministério da Saúde de comprar imunobiológicos de produtores nacionais ou via produtor nacional, também facilitou estes 
acordos de transferência tecnológica, pois permitiu, de certa maneira, o acesso do mercado nacional à empresa multinacional detentora da tecnologia. Nestes casos, tanto a empresa receptora como a empresa fornecedora da tecnologia são beneficiadas na parceria (win-win partnership).

As características da transferência para a produção da vacina de influenza pelo Instituto Butantan podem ser assim resumidas: de uma empresa privada com fins lucrativos para um produtor público; seu preço não foi explicitado, nem pago separadamente, ficando embutido na venda do produto a granel no periodo; e acordo formal de assistência técnica entre Fundação Butantan e Sanofis-Pasteur para obtenção de um contrato de licença de tecnologia.

As questões relativas ao "porquê" da transferência explicitadas na metodologia deste artigo foram vistas de forma a identificar os aspectos envolvidos na tomada de decisão, além da relevância e oportunidade de criação de conhecimento tecnológico por parte do setor público na produção da vacina de influenza. Também se destaca a análise das pré-condições, por parte do Instituto Butantan, na incorporação dessa tecnologia, além de sua importância no contexto internacional (Organização Mundial da Saúde/OMS) na avaliação e criação de condições para o controle da epidemia sazonal de influenza e possiveis pandemias. Por fim, fatores que influenciaram a decisão do Ministério da Saúde (MS) na incorporação da vacina de influenza no PNI por razões epidemiológicas e políticas também foram consideradas.

As questões relativas ao "como", ou seja, ao processo em si da transferência, objetivaram identificar e analisar: os instrumentos formais (contrato de transferência de tecnologia), as etapas de implementação, transferência, fatores intervenientes, relações entre os parceiros - Sanofis Pasteur e IBu, entraves institucionais, impactos econômicos e, por fim, discutir os resultados e as possibilidades de inovação criadas por esse processo.

Para fins de sistematização do estudo, foi feita uma periodização do processo e seu desenvolvimento, levando em consideração os contextos internos e externos:

10 Período (de 1999 a 2004): Tomada de decisão no processo de transferência, bases legais, acordos e início 
7.

WHO. Genebra, 2005. da primeira fase de implementação - construção da fábrica e compra de equipamentos;

2。 Período (de 2005 a 2008): Término das instalações civis e início da transferência propriamente dita;

3० Periodo (de 2009 a 2011): Última fase - certificação da transferência.

\section{Análise dos períodos}

\subsection{Primeiro Período (de 1999 a 2004). Tomada de decisão e início do processo de transferência}

A análise da relevância dessa transferência parte de dois eixos centrais: a vertente epidemiológica e sanitária e a vertente da autossuficiência no setor produtivo. A decisão, tanto de vacinar toda a população idosa contra influenza quanto a de definir sua produção por um instituto público dentro de uma linha do Plano Nacional de Autossuficiência, são os fatores determinantes para a tomada de decisão apontados nas entrevistas realizadas. Do ponto de vista do cenário internacional, destaca-se o fato do Instituto ser o primeiro produtor dessa vacina no hemisfério sul, criando a possibilidade de atender demandas para além do nivel nacional. Além disso, a experiência passada relacionada à questão da gripe espanhola mostrou que só seria possivel a produção de vacinas contra gripes pandêmicas se houvesse no país uma estrutura de produção da vacina contra a gripe sazonal. Assim, a produção da vacina da gripe sazonal faz parte da preparação do IBu contra surtos futuros de gripe pandêmica.

\subsubsection{Tomada de decisão para a transferência}

No contexto internacional, o papel desempenhado pela OMS foi um dos fatores centrais na escolha da vacina de influenza: provavelmente a única doença infecciosa que a OMS cuida com certa profundidade e eficácia... no fundo quem desenvolve a vacina é a WHO e disponibiliza para o mundo inteiro, gratuitamente. Quer dizer o que o fabricante tem de fazer é saber pegar a vacina e produzir (RAW, 2011).

No contexto nacional, dois atores institucionais foram centrais para a tomada de decisão: o Ministério 
Charles Merieux (1907-2001) foi um médico especialista em sorologia formado pelo Instituto Pasteur. Entre suas atividades como cientista está a introdução da cultura de células "in vitro" na produção de vacinas. Em 1967 criou a Fundação Marcel Merieux, uma associação sem fins lucrativos cujo propósito era contribuir para a pesquisa. 0 Instituto criado mais tarde, em 1989, vincula-se ao Instituto Pasteur dando origem à empresa Pasteur-Merieux Sérums et Vaccins, mais tarde renomeada para Sanofi Pasteur (Azevedo, 2007). da Saúde e o Instituto Butantan. Também apareceriam no processo de implementação a Secretaria de Estado da Saúde e a Sanofi-Pasteur (à época, Sanofis-Aventis).

A decisão do MS de incluir no PNI a vacinação dos idosos pelo Brasil criou duas novas situações: a necessidade de alterar rotinas desse programa, levando a incorporação de outra faixa de idade às Unidades Sanitárias, e a necessidade de fornecimento ou produção desta vacina pelo mercado internacional ou nacional.

Já em relação ao tipo de transferência a ser realizada e à escolha da parceira, o IBu teve papel central. Neste sentido, as diferentes falas dos entrevistados e trabalhos anteriores produzidos pelas lideranças cientificas da instituição, em especial, de Isaias Raw (2011), é posta uma questão central na transferência que é a definição dos termos desse processo. Esta deveria ser feita na imagem de um pacote aberto, onde quem iria definir a planta, o tamanho, a capacidade de produção seria o próprio IBu, reafirmando a capacidade deste de produzir, desenvolver e incorporar essa tecnologia intermediada pela sua Fundação de apoio. (MERCADANTE, 2011 e CHAIMOVICH, 2011)

A respeito da parceira, a escolha da multinacional Sanofi Pasteur é avaliada da seguinte maneira por Raw:

"Por que a Sanofi? Porque eu conhecia bem o velho Mérieux $x^{8}$ enquanto eu estava fora do país... Ele tinha uma visão social muito importante. Então, eu me entendi com ele e disse eu quero a tecnologia da vacina da gripe por uma razão muito simples, porque se eu disser que eu estou fazendo igual à Aventis, não tem mais discussão, porque eu estou fazendo de acordo com a Comunidade Europeia. Enfim, o Mérieux concordou em passar a tecnologia. Número dois, nós não tínhamos dinheiro, então foi negociada uma coisa sui generis, que depois a Fiocruz copiou em outras condições. Quer dizer o seguinte: eu, enquanto não tenho a fábrica, eu compro a vacina, e no preço da vacina você embute a transferência da tecnologia. Portanto nós vamos pagar por vacina comprada e não por tecnologia... Ficou definido que enquanto nós não fabricamos, nós podemos comprar, com prioridade" (RAW 2011). 
De fato, a figura de Isaías Raw foi fundamental na formulação do projeto, na proposta apresentada ao Ministério da Saúde, e na estratégia de desenvolvimento da vacina em parceria com a empresa Sanofis-Pasteur.

Um dos questionamentos do estudo foi referente ao tempo de viabilidade ou de breve obsolescência da tecnologia transferida ou o risco de comprar uma tecnologia já ultrapassada. As respostas não descartam a possibilidade de outras tecnologias surgirem para a confecção da Vacina Influenza, mas questionam a trajetória, o tempo e o custo de uma nova produção frente a esse processo, e defendem a validade da transferência dessa tecnologia pelas condições existentes no próprio IBu, que já dominava essa tecnologia em menor escala, e o cenário nacional e internacional. Assim, desenha-se uma equação onde se avalia as diferentes variáveis, o custo-benefício das distintas tecnologias, o custo de produção, a capacidade produtiva, os problemas existentes para cada uma das tecnologias, os insumos empregados e o acesso a eles, os equipamentos, as áreas e as infraestruturas necessárias, dentre outras variáveis.

\subsubsection{Instrumento formal da transferência}

0 Contrato de Assistência Técnica para a realização da transferência foi assinado em primeiro de outubro de 1999, sendo a então Pasteur Merieux Sérums et Vaccins, representada por Michel Greco, Diretor Presidente, e a Fundação Butantan, representada por seu Presidente, Isaías Raw, atuando pelo Instituto Butantan. Ainda assinam como testemunhas: José da Silva Guedes, Secretário de Estado da Saúde, e Jarbas Barbosa da Silva Júnior, Diretor do CENEPI (FUNASA). (TECHNOLOGY LICENSE AGREEMENT 1999)

A definição de Assistência Técnica Central especifica os seguintes itens: utilização da vacina trivalente final a granel, e o controle de qualidade respectivo; a mistura da Vacina Influenza monovalente, e o controle de qualidade relativo; produção de vacina influenza a granel, e o controle de qualidade relativo; organização e controle do sistema de ovos; fabricação de rotina do lote de sementes operacionais atuais, de acordo com as recomendações da OMS (Organização Mundial de Saúde - WHO 2005). 
A previsão era que o contrato duraria quatro anos, com vencimento previsto para fevereiro de 2004, podendo ser encerrado antes, conforme estabelecido no instrumento, assim que se desse a total conclusão da assistência técnica.

Do ponto de vista da tecnologia de fabricação, esta envolvia, pelo contrato, o acompanhamento das etapas de construção das edificações, suprimentos, equipamentos e processos. A transferência de cada um destes itens seria consubstanciada pelo suprimento de documentação (forma tangivel da transferência de tecnologia), treinamento e qualificação e a validação, que deveria ser considerada como assistência técnica, era diretamente relacionada à especificidade da tecnologia de produção da Vacina Influenza e parte da transferência de tecnologia.

Por fim, para cada etapa do processo, a validação seria atingida através da produção de três lotes do produto que atendessem às especificações correspondentes.

\subsubsection{A construção da fábrica}

A primeira etapa do processo de planejamento e construção das instalações e aquisição de equipamentos, sob responsabilidade do Butantan, teve aporte financeiro da Secretaria de Estado da Saúde e do Ministério da Saúde, sendo Isaías Raw fundamental na obtenção dos recursos.

As tabelas a seguir resumem os aportes financeiros contratuais destinados à construção da fábrica e compra de equipamentos. 


\begin{tabular}{|c|c|c|c|}
\hline \multicolumn{4}{|c|}{$\begin{array}{l}\text { Recursos financeiros contratuais destinados à construção e compra de } \\
\text { equipamentos para a Fábrica de Influenza no periodo de 2003-2007 }\end{array}$} \\
\hline \multicolumn{2}{|r|}{ Formas Contratuais } & Recursos em reais & $\begin{array}{l}\text { Equivalência } \\
\text { aproximada } \\
\text { em dólares }\end{array}$ \\
\hline \multirow{5}{*}{ Obras civis } & Contrato Inicial & $\mathrm{R} \$ 17.253 .698,00$ & US\$8.216.046,67 \\
\hline & $1^{\circ}$ termo aditivo & $\mathrm{R} \$ 5.243 .500,00$ & US $\$ 2.496 .904,76$ \\
\hline & $2^{\circ}$ termo aditivo & $\mathrm{R} \$ 3.379 .522,00$ & US\$1.609.296,19 \\
\hline & Reajuste do contrato inicial & $\mathrm{R} \$ 1.537 .268,00$ & US\$732.032,38 \\
\hline & Subtotal: obras civis & $\mathrm{R} \$ 27.413 .988,00$ & US\$13.054.280,00 \\
\hline \multirow{3}{*}{ Equipamentos } & $\begin{array}{l}\text { Convênio Ministério da } \\
\text { Saúde } 57 / 2003\end{array}$ & $\mathrm{R} \$ 34.463 .459,84$ & US\$16.411.171,30 \\
\hline & $\begin{array}{l}\text { Convênio Ministério da } \\
\text { Saúde } 2242 / 2003\end{array}$ & $\mathrm{R} \$ 14.426 .865,00$ & US\$6.869.935,71 \\
\hline & Subtotal: equipamentos & $\mathrm{R} \$ 48.890 .324,84$ & US\$23.281.107,06 \\
\hline \multicolumn{2}{|l|}{ Total geral } & $\mathrm{R} \$ 76.304 .312,84$ & US $\$ 36.335 .387,05$ \\
\hline
\end{tabular}

Fonte: Departamento de Obras da SES-SP e Portal da Transparência do Governo Federal: http://www. portaltransparencia.gov.br/
0 contrato inicial foi de $\mathrm{R} \$ 17.253 .698,00$, tendo um $1^{\circ}$ termo aditivo no valor de $\mathrm{R} \$ 5.243 .500,00$, um $2^{\circ}$ termo aditivo de $\mathrm{R} \$ 3.379 .522,00$ e um reajuste no valor inicial de $R \$ 1.537 .268,00$, totalizando $R \$ 27.413 .892,00$.

A construção da fábrica subsidiada pelo Governo do Estado de São Paulo, por meio da Secretaria de Estado da Saúde, teve sua licitação realizada no ano de 2003 e sofreu uma impugnação em 2004, tendo suas obras iniciadas somente em abril de 2005, com previsão de término para 540 dias.

A obra foi entregue em 14 de maio de 2007, totalizando 760 dias para sua construção. Mercadante refere que: "Acho que ficou mais de um ano para a discussão de julgamento, recurso e tudo mais. Recursos 
das construtoras que entraram na competição, certo? $\mathrm{E}$ é interessante, acho que vale a pena reforçar isso, pensando nesses 10 anos teve também dificuldades do ponto de vista tecnológico, porque não é simples essa incorporação, mas acho que o problema principal foi formal, legal". (MERCADANTE, 2011)

A compra dos equipamentos foi subsidiada pelo Ministério da Saúde por meio dos convênios 57/2003 e 2242/2003, visando a aquisição e instalação de equipamentos para produção da vacina contra a influenza. No primeiro foram destinados à Fundação Butantan $\mathrm{R} \$ 34.463 .459,84$ e no segundo, foram destinados $R \$$ $14.426 .865,00$, totalizando $\mathrm{R} \$ 48.890 .324,84$.

\subsection{Segundo Período (de 2005 a 2011). Início da transferência propriamente dita}

Ao longo da transferência tecnológica, as pandemias de gripe aviária H5N1 (2005) e suína AH1N1 (2009) alteraram o processo de implementação, modificando a fábrica para que atendesse à demanda dessas novas cepas.

A criação a partir de 2005 de uma planta piloto para o desenvolvimento de 20.000 doses de vacina em caráter emergencial foi visto como um fator importante, não só para fixar o domínio da tecnologia em pequena escala, mas também para definir, do ponto de vista institucional, condições para o passo maior que era de instalação da fábrica.

\subsubsection{0 episódio Gripe Aviária}

0 episódio da gripe aviária foi visto pelos diferentes atores chaves como uma oportunidade de implementação e desenvolvimento por parte do $\mathrm{IBu}$, para a confecção da vacina influenza, de certa forma condicionada pelo processo em curso, mas que fugia das regras explicitadas na transferência com a Sanofi. A decisão de construção de uma planta piloto para a fabricação em pequena escala foi em comum acordo com o Ministério da Saúde e teve 0 apoio da OMS. 
0 Ministério da Saúde por meio do convênio 1607/2005MS, referente ao "Projeto para Produção da Vacina contra a Influenza CEPA H5N1 (conhecida como gripe aviária)", transferiu $\mathrm{R} \$ 3.124 .000,00$ para a Fundação Butantan. Esses recursos foram destinados à construção de uma planta piloto para estudos e produção em pequena escala de uma vacina contra uma eventual epidemia.

A necessidade de implantar a planta piloto agilizou parte dos procedimentos da transferência, incluindo o treinamento, checagem de instalações, equipamentos e 0 fornecimento de ovos. Os treinamentos tiveram início em 2005, com a ida de técnicos do IBu para a sede da Sanofi na França, como relata a pesquisadora responsável Cosue Miyaki: "Em 2007 a gente começou a produção, mas não com $\mathrm{H} 5$, para aprender com outro vírus que eles nos forneceram e somente em 2007, a gente começou a mexer com a H5N1". (MIIAKI, 2011)

A implantação da planta piloto do H5N1 não estava no escopo do contrato de transferência e permitiu fugir um pouco das regras estabelecidas pela Sanofi e experimentar, em pequena escala, algumas modificações do processo. Outra questão importante dessa experiência foi que a equipe local constituída para a planta piloto foi treinada como relata Cosue "(...) os mesmos funcionários que trabalharam comigo no $\mathrm{H} 5$ foram pra lá, eles são atualmente os coordenadores de cada área, eles na verdade que comandam a produção, cada um com a sua equipe" (MIYAKI, 2011).

\subsubsection{Recursos humanos}

No segundo semestre de 2005 foi realizado um concurso público para contratação de 96 funcionários estatutários para trabalharem na fábrica de influenza. 0 concurso apresentou problemas, a anuência e posse aconteceram um ano depois, em junho e outubro, respectivamente. 0 fato da fábrica não estar pronta fez com que esses funcionários fossem alocados em outras seções da Divisão de Produção. A incorporação destes, adquirindo experiência em outros processos produtivos, criou ao final problemas para algumas seções na hora de transferi-los de volta à 
fábrica. Um outro problema apontado foi o conflito entre as expectativas dos candidatos aprovados no concurso e as da administração, já que grande parte desses candidatos esperava atuar em seções da Divisão Científica do Instituto.

0 resultado desses impasses iniciais foi que de todos os funcionários que assumiram suas funções no período de vigência do concurso, apenas 35 voltaram para a fábrica de Vacina Influenza e desses muitos saíram em seguida da fábrica. A maioria dos funcionários que atuou diretamente na fábrica entre 2007 e 2008, foi transferida para laboratórios da Divisão Científica e Centro de Desenvolvimento Cultural do Instituto.

A solução encontrada foi a substituição de funcionários estatutários da fábrica, no final de 2008, por funcionários contratados pela Fundação Butantan como celetistas e a incorporação de funcionários estatutários já concursados pelo Instituto Butantan anteriormente.

\subsubsection{A implementação propriamente dita}

Uma primeira constatação é que o processo de transferência de tecnologia em si e o treinamento de produção foram rápidos - cerca de dois anos após o término das obras civis e a chegada dos equipamentos, como informa Cosue Miyaki:

se for contar, o prédio que está sendo construído eu não sei desde quando, mas se a gente for contar o treinamento do pessoal recebendo ovo que começou mais em 2008, 2009 e 2010, foi rapidissimo, dois anos (MIYAKI, 2011).

As etapas de implementação da transferência podem ser assim resumidas: a primeira foi a compra da Vacina Influenza junto à Sanofis Pasteur, a segunda foi a formulação e envase dessas vacinas vindas em concentrados fornecidos pela parceira, e a última etapa foi a produção completa da vacina em toda a sua cadeia pelo Butantan.

$\mathrm{Na}$ descrição de Cosue Miyaki, o processo envolveu os seguintes passos: 
a) A primeira etapa foi capacitar o pessoal para os exames de controle do produto final. Para isso a equipe foi treinada na fábrica na França, onde ficou o tempo necessário para incorporar toda a tecnologia, que não é trivial e trazer para o Butantan. 0 Butantan comprava o produto pronto, embalado da Sanofis e aqui se faziam os testes finais de liberação.

b) A segunda etapa foi a de formulação. Como a vacina da gripe é composta por três vírus diferentes, são três vacinas diferentes e vinham os três produtos a granel. No Butantan a equipe desenvolveu competência para misturar. Era preciso examinar cada uma como se fosse uma vacina diferente, fazer o controle, a concentração e a capacidade de cada uma e misturar de forma que a formulação feita tenha eficácia e proteção.

c) Isso tudo foi feito até que a fábrica ficou pronta (instalação física), se definiu a instalação dos equipamentos, tudo monitorado rigorosamente pela Sanofis.

Ainda ao final do periodo outro problema interveniente nessa etapa do processo foi o aparecimento do vírus H1N1 (gripe suína) que colocou, além das questões previstas, outras, como um reforço maior na área de biossegurança." (MIYAKI, 2011).

É importante destacar o papel fundamental que teve Hernan Chaimovich, superintendente da Fundação Butantan à época, principalmente pela solicitação e autorização de todas as ações necessárias para o início das atividades fabris da planta. Para a execução dessas ações de produção dos monovalentes de influenza, foram fundamentais as participações de Cosue Miyaki e de Maurício Meros de Oliveira.

\subsection{Terceiro Período (de 2009 a 2011). Certificação da transferência.}

Neste periodo, se implementaram todas as etapas de desenvolvimento da vacina no IBu (2008/09), o início das operações na fábrica (2010) e a certificação em 2011. 0 resumo das etapas que se seguiram:

começou pelo recebimento dos ovos na escala prevista (123.840) e então deu-se o startup na fábrica em 2010, em setembro, então a fábrica passou a funcionar, mas sem 
vírus, só mexendo com a quantidade de um lote de ovos. Em 2010 a gente começou a manipulação com vírus, eles vieram novamente pra ver se estava tudo ok... Foi em setembro de 2010 mais ou menos, ou antes, junho-julho, e ai a gente começou a produção (MIYAKI, 2011).

Apesar da certificação obtida pela Sanofi Pasteur, a produção própria do IBu não estava atendendo ainda às questões regulatórias para a fabricação final da vacina exigidas pela ANVISA.

\subsubsection{Certificação pela ANVISA}

A certificação pela ANVISA envolveu não somente a fábrica da Influenza, mas o conjunto de instalações e interdependências do setor produtivo do IBu. As questões estruturais ainda constituíram entraves e precisaram ser modificadas e adequadas ao novo padrão de qualidade. Essa questão mobilizou os dirigentes do Butantan e as mudanças propostas foram implementadas. A Diretora de Produção à época, assim se referia a esta relação:

A relação hoje Instituto Butantan/ANVISA é boa, ela é bem melhor do que nós tivemos no passado... Eu tenho o seguinte entendimento, as resoluções ou as exigências elas significam a melhoria contínua, então a cada momento eles estreitam mais, têm mais exigências, porque as exigências do Butantan são as mesmas pra indústria farmacêutica, não há um entendimento de que porque nós somos um órgão público nós temos que ser diferentes, não é. Pelo contrário, a ANVISA entende, eu também acho, nós devíamos ser o exemplo, nós tínhamos que ser o exemplo. (PRADO, 2012).

A certificação de Boas Práticas de Produção (BPF) pela ANVISA foi uma etapa regulatória fundamental para 0 início da produção da vacina sazonal da influenza. 0 Diretor do Instituto à época, Jorge Kalil, foi central neste processo, autorizando todas as ações necessárias para a obtenção desta certificação. Aliás, foi na sua gestão que foram obtidas as atuais certificações das áreas fabris do Instituto Butantan até a presente data, da área de Formulação, Envase e Embalagem, da área de Produção dos Monovalentes da Influenza e também, da área de 
Processamento de Plasma Hiper imune Equino. As duas primeiras certificações permitiram a produção da vacina de influenza em condições de BPF em toda a sua cadeia de produção, enquanto a certificação da última área, juntamente com a área de Formulação, Envase e Embalagem, permitiu a produção de imunoglobulinas equinas ("soros") para tratamento de envenenamentos ofídicos, de aranhas, escorpiões, lagartas, toxinas e por vírus rábico, em toda a sua cadeia de produção. Para a execução das ações necessárias para a obtenção da certificação BPF, foram fundamentais as coordenações de Cláudio Cabral, Diretor da área de Regulatórios, e de Paulo Lee Ho, Diretor da Divisão de Desenvolvimento Tecnológico e de Produção do Instituto Butantan.

A abrangência do material recolhido pelo estudo aponta, na análise desse processo, algumas questões que julgamos relevantes e serão apresentadas nas considerações finais.

\section{Considerações finais}

0 estudo em questão mostra de maneira singular a complexidade de um processo de transferência tecnológica ocorrido entre o IBu e a empresa privada Sanofis Pasteur e aponta para fatores intervenientes de diferentes naturezas na sua realização enquanto estratégia de autossuficiência do setor produtivo público.

Sem entrar em juizo de valor, pode-se dizer que uma série de argumentos aqui apresentados justificam a fabricação nacional da Vacina Influenza, assim como justificam a produção dessa vacina dentro de uma instituição pública de produção e de pesquisa, potencializando processos de inovação em novas tecnologias e/ou vacinas.

Do ponto de vista institucional, o estudo apresenta, de um lado, a possibilidade de levar adiante esse processo amparado pelas pré-condições encontradas, mas, de certa forma, aponta para fragilidades, sejam das estratégias políticas de CT\&l em curso no apoio às instituições públicas, sejam na estrutura em si destas instituições. Neste caso, o Instituto Butantan e seus limites dados por entraves estruturais sérios para seu desenvolvimento. 
As repercussões do ponto de vista econômico e financeiro mereceriam um estudo mais aprofundado, levando em consideração a balança comercial e outras questões relativas, os gastos realizados com a compra de vacinas e investimentos no periodo compreendido de 12 anos num montante de aproximadamente 2 bilhões de reais.

Os dados e evidências desse estudo também trazem à tona uma série de situações positivas e possibilidades de ganhos com a produção local e seu potencial inovador. $\mathrm{Na}$ área social, pelo custo-benefício do ponto de vista da economia que se faz com a diminuição das internações e dos medicamentos necessários para tratamento das pessoas contaminadas pela gripe.

Por outro lado, a velocidade de uma transferência de tecnologia é uma questão problemática a ser resolvida. São planejamentos de longo prazo que muitas vezes cobrem vários governos e por isto, devem ser considerados estratégicos e prioridades de Estado e não de governos.

Além disso, existe um conflito a ser resolvido relacionado com a questão de compras pelo Estado. Muitos dos equipamentos e processos são muito específicos e não existe concorrência no mercado, sendo muito difícil a contratação, compra de serviços e equipamentos pela Portaria 8666 de 1993 em processos licitatórios, que levam muito tempo e são passiveis de judicialização. Estas questões acabam impactando na velocidade de uma transferência de tecnologia, sendo que este cenário praticamente inexiste no setor privado, tornando o processo atual de transferência de tecnologia menos competitivo do que se deseja no setor público. 


\section{Referências}

\section{Entrevistas realizadas}

CCHAIMOVICH, Hernan. Entrevista concedida a Nelson Ibañez e Olga Sofia Fabergé Alves para o Projeto "PPSUS - Transferência de tecnologia da vacina de influenza". São Paulo, 11/04/2011, transcrição Sônia Claro.

MANCINI, Dalva. Entrevista concedida a Carlos Eduardo Dias e Olga Sofia Fabergé Alves para o Projeto Ciência e Cientistas do Instituto Butantan. São Paulo, 21/08/2014, transcrição CEALAG.

MEROS, Maurício. Entrevista concedida a Nelson Ibañez e Suzana César Gouveia Fernandes para o Projeto "PPSUS - Transferência de tecnologia da vacina de influenza". São Paulo, 20/04/2012, transcrição Sônia Claro.

MERCADANTE, Otávio Azevedo. Entrevista concedida a Nelson Ibañez, Maria Cristina da Costa Marques e Suzana César Gouveia Fernandes para o Projeto "PPSUS - Transferência de tecnologia da vacina de influenza". São Paulo, 17/03/2011, transcrição Sônia Claro.

MIYAKI, Cosue. Entrevista concedida a Nelson Ibañez, Olga Sofia Fabergé Alves e Suzana César Gouveia Fernandes para o Projeto "PPSUS - Transferência de tecnologia da vacina de influenza". São Paulo, 29/03/2011, transcrição Sônia Claro.

PRAD0, Sally Müller. Entrevista concedida a Nelson Ibañez e Olga Sofia Fabergé Alves para o Projeto "PPSUS Transferência de tecnologia da vacina de influenza". São Paulo, 25/04/2012, transcrição Sônia Claro.

RAW, Isaías. Entrevista concedida a Nelson Ibañez e Olga Sofia Fabergé Alves para o Projeto "PPSUS Transferência de tecnologia da vacina de influenza". São Paulo, 28/04/2011, transcrição Flávia Urzua. 


\section{Referências Bibliográficas}

ACOSTA, S. . Vital Brazil e o caso da Casa Armbrust, as relações entre a ciência e os cientistas e a gestão em saúde pública. Cadernos de História da Ciência, v. 10, p. 55-76, 2015.

ARANTES, Altino. Meu diário - registro íntimo de fatos e de impressões. Arquivo do Estado de São Paulo (Aesp). Arquivo Privado de Altino Arantes (Apaa) lócus: AP91.01.001.

BERTOLLI, C. A Gripe Espanhola em São Paulo, 1918: Epidemia e Sociedade. São Paulo: Editora Paz e Terra, 2003.

BERTUCCI-MARTINS, L. Influenza, a medicina enferma: Ciência e práticas de cura na época da gripe espanhola em São Paulo. 2002. Tese (Doutorado em História) - Instituto de Filosofia e Ciências Humanas, Universidade Estadual de Campinas, Campinas.

BRASIL. Controladoria-Geral da União. Produção, pelo convenente, dos imunobiológicos que integram - Programa Nacional de Imunizações, desenvolvido pelo Ministério da Saúde, na forma a seguir descrita: 33.900 .000 doses de Vacina de influenza. Publicado no Diário Oficial da União em: 05/04/2012.

BRASIL. Ministério da Saúde. Campanha nacional de vacinação contra gripe 2012 de 05/05/2012 a 25/05/2012. Coberturas vacinais total Brasil. SI-PNI - Sistema de Informação do Programa Nacional de Imunizações, 2012.

BRASIL. Controladoria-Geral da União. Produção de imunobiológicos necessários às ações do Programa Nacional de Imunizações na forma a seguir descrita: vacina contra influenza: 32.750 .000 doses. Publicado no Diário Oficial da União em: 19/01/2011.

BRASIL. Ministério da Saúde. Campanha nacional de vacinação contra gripe 2011. Iniciada em de 25/04/2011 a 13/05/2011. Coberturas vacinais totais Brasil. SI-PNI - Sistema de Informação do Programa Nacional de Imunizações, 2011. 
BRASIL. Controladoria-Geral da União. Aquisição e instalação de equipamentos, visando a produção da vacina contra Influenza. Publicado no Diário Oficial da União em: 26/12/2003. Consultado em: 10/06/2012.

BRASIL. Controladoria-Geral da União. Aquisição de Imunobiológicos Contra Influenza. Publicado no Diário Oficial da União em: 08/01/2004.

BRASIL. Portal da Transparência. CEPA H5N1 - Planta Piloto 2005. Disponivel em: http://www.portaltransparencia.gov.br/convenios/

CHAIMOVICH, H. Ciência, Tecnologia e Produção no Instituto Butantan. Revista USP. São Paulo, n.89, p. 78-89, março/maio 2011.

FERNANDES, S.C.G. O Instituto Butantan entre 1928 e 48: uma visão sócio-cultural de sua organização interna. Tese de Doutorado apresentada em 2008 na Faculdade de Filosofia, Letras e Ciências Humanas da Universidade de São Paulo, USP, 2011.

GADELHA, C.A.G. A produção e o desenvolvimento de vacinas no Brasil. Hist. cienc. saúde-Manguinhos. Rio de Janeiro, v. 3, n. 1, p. 111-132, Jun 1996. .

HO, P.L., MIYAJI, E.N., OLIVEIRA, M.L.S., DIAS, W.O., KUBRUSLY, F.S., et al. (2011) Economical Value of Vaccines for the Developing Countries-The Case of Instituto Butantan, a Public Institution in Brazil. PLoS Negl Trop Dis 5(11): e1300.

IBAÑEZ, N., FERNANDES, S.C.G.; FARIA, M., WEN, FH., SANT'ANNA, AO. De Instituto Soroterápico a Centro de Medicina Experimental: institucionalização do Butantan no período de 1920 a 1940. In: Cadernos de História da Ciência, São Paulo, vol. 2, n 2, janeiro/junho de 2006.

IBAÑEZ, N., WEN, FH., FERNANDES, SCG. A autossuficiência na produção de imunobiológicos e a criação do Centro de Biotecnologia do Instituto Butantan. In: Cadernos de História da Ciência. São Paulo, vol. 3, no 1, janeiro/junho de 2007.

INSTITUTO BUTANTAN. Relatórios de Gestão do Instituto Butantan: 1918 e 1948. Acervo: Centro de Memória do Instituto Butantan.

INSTITUTO BUTANTAN. Technology License Agreement. 1999. Acervo do Cedoc. 
MELO, L.M. "Sistema Nacional de Inovação (SNI): uma proposta de abordagem teórica", Texto para Discussão, IE-UFRJ, n.357, abr., 1996.

MEYER, C. L.; TEIXEIRA, J. R. A grippe epidemica no Brazil e especialmente em São Paulo. São Paulo: Casa Duprat, 1920.

O ESTADO DE SÃO PAULO. São Paulo, 27 out. 1918. Disponivel em: https://acervo.estadao.com.br/ pagina/\#!/19181027-14547-nac-0003-999-3-not

RAW, I. entrevista in "Década de 80: 0 programa de auto suficiência em imunobiológicos e o SUS". Cadernos de História da Ciência. São Paulo, vol. 3, n 1, janeiro/junho de 2007

SEIDL, C.P. A propósito da pandemia de gripe de 1918: argumentos e fatos irrespondíveis. Rio de Janeiro: 1919. Bernard Frères.

VIANA, A. L. d'Á et al. Inovação e Saúde: Capacidade regulatória público-privada em dois processos tecnológicos: 1. Organizações Sociais de Saúde (OSS); 2. Tomografia Computadorizada por Emissão de Pósitrons (PET-CT). Relatório Final de Pesquisa: Convênio FAPESP-CNPq-SUS (PP-SUS 2006/2007). São Paulo, setembro de 2009, 271p.

VIANA, A. L. d'Á; BOUSOUAT, A.; IBAÑEZ, N. (orgs.). Saúde, desenvolvimento, ciência, tecnologia e inovação. São Paulo: Hucitec - Cealag, 2012

WORLD HEALTH ORGANIZATION. Plan Mundial de la OMS de Preparación para una Pandemia de Influenza. WHO, Geneva, 2005. Sítios Eletrônicos Consultados: www.abdi.com.br www.bndes.gov.br www.saude.gov.br

YIN, RK. Case Study Research - Design and Methods. Newbury Park: Sage; 1994. 


\section{Bibliografia consultada}

ALBUQUERQUE E.M.; CASSIOLATO J.E. As especificidades do sistema de inovação do setor saúde: uma resenha da literatura como introdução a uma discussão sobre o caso brasileiro. Belo Horizonte: Fesbe; 2000.

ALBUQUERQUE EM. National systems of innovation and non-OECD countries: notes about a tentative typology. Revista de Economia Política, v. 19, n. 4, p. 35-52, 1999.

ALMEIDA, Marta de. República dos invisíveis: Emílio Ribas, microbiologia, e saúde pública em São Paulo 18981917. Bragança Paulista: editora da Universidade São Francisco, 2003.

ARAÚJO, José Duarte de. "Saúde e desenvolvimento econômico: atualização de um tema". Rev. Saúde Pública, v.9, n.4, p.515-528, Dez 1975. BENCHIMOL JL, TEIXEIRA LA. Cobras, lagartos e outros bichos: Uma história comparada dos Institutos Oswaldo Cruz e Butantan. Rio de Janeiro: UFRJ, 1993.

BERTOLLI, C. A Gripe Espanhola em São Paulo, 1918: Epidemia e Sociedade. São Paulo: Editora Paz e Terra, 2003

BRAZIL, V. Memória Histórica do Instituto Butantan. São Paulo: Elvino Pocai, 1941.

CAMARGO, A. M. F. Os impasses da pesquisa microbiológica e as políticas de saúde pública em São Paulo (1892-1934). 2004. Dissertação (Mestrado) Unicamp, Campinas, 2004.

CAMARGO, EP.; SANT'ANNA, 0. A. Institutos de pesquisa em saúde. Ciência \& Saúde Coletiva, v. 9, n. 2, p. 295, abril/jun. 2004.

CAMMAROTA, F. C. Fundação Estatal: fundamentos legais e institucionais. Brasília: Secretaria de Gestão do Ministério do Planejamento, Orçamento e Gestão. 2005.

CAPANEMA, L. X. L.; PALMEIRA FILHO, P. L.; PIERONI, J. P. Apoio do BNDES a Complexo Industrial da Saúde: experiência do Profarma e seus desdobramentos. BNDES Setorial, Rio de Janeiro, n. 27, 2008. 
CASSIOLATO, J. E; LASTRES, H. M. M. Inovação e sistemas de inovação: relevância para a saúde. RECIIS - R. Eletr. De Com. Inf. Inov. Saúde, Rio de Janeiro, v. 1, n. 1, p.153-162. 206, jan./jun. 2007,

CHAIMOVICH, Hernan. Ciência, Tecnologia e Produção no Instituto Butantan. Revista USP. São Paulo, n.89, p. 78-89, mar./mai. 2011.

CHANG, Ha-Joon. Chutando a escada. A estratégia do desenvolvimento em perspectiva histórica. São Paulo: Unesp, 2002.

CHANG, Ha-Joon. Rethinking development economics. London: Anthem Press, 2003.

CHAVES CV, Albuquerque EM. Desconexão no sistema de inovação no setor saúde: uma avaliação preliminar do caso brasileiro a partir de estatísticas de patentes e artigos. Econ. Aplicada , v. 10, n. 4, p. 523539, 2006

CORDEIRO, Hésio. A indústria da saúde no Brasil. Rio de Janeiro: Graal, 2a edição, 1985.

CRUZ, Carlos Henrique de Brito. Ciência, Tecnologia e Inovação no Brasil: desafios para o período 2011 a 2015. Revista Interesse Nacional, v. 10, n. 3,p. 7591, jul./ aset. 2010.

DAGNINO, R. Ciência e tecnologia no Brasil: o processo decisório e a comunidade de pesquisa. Campinas: Editora da Unicamp, 2007.

DONALISIO, Maria Rita; FRANCISCO, Priscila Maria Stolses Bergamo; LATORRE, Maria do Rosário Dias de Oliveira. Tendência da mortalidade por doenças respiratórias em idosos antes e depois das campanhas de vacinação contra 207 influenza no Estado de São Paulo - 1980 a 2004. Rev. bras. epidemiol., São Paulo, v. 9, n. 1, Mar. 2006.

FERNANDES, S.C.G. 0 Instituto Butantan entre 1928 e 48: uma visão sócio-cultural de sua organização interna. 2008. Tese (Doutorado) - Faculdade de Filosofia, Letras e Ciências Humanas da Universidade de São Paulo, São Paulo, 2011.

FRANCISCO, Priscila Maria Stolses Bergamo; DONALISIO, Maria Rita; LATTORRE, Maria do Rosário Dias de Oliveira. Internações por doenças respiratórias em idosos e a intervenção vacinal contra influenza no Estado de São Paulo. Rev. bras. epidemiol., São 
Paulo, v. 7, n. 2, Jun. 2004. Disponível em: http:// dx.doi.org/10.1590/S1415-790X2004000200011. Acesso em: 04 Jul. 2012..

GADELHA, C. A. G. Biotecnologia em saúde: um estudo da mudança tecnológica na indústria farmacêutica e das perspectivas de seu desenvolvimento no Brasil. 1990. 365 f. Dissertação (Mestrado em economia) - Instituto de economia, Universidade estadual de Campinas, Campinas, 1990.

GADELHA, C. A. G. Desenvolvimento, complexo industrial da saúde e política industrial. EM: Rev. Saúde Pública, São Paulo, v. 40, n. spe, 2006.

GADELHA, C. A. G. 0 complexo industrial da saúde e a necessidade de um enfoque dinâmico na economia da saúde. Ciência \& Saúde Coletiva, Rio de janeiro, v. 8, n. 2, p=p.521-535, 2003.

GADELHA, C. A. G.; MALDONADO, J. M. D. V. 0 papel da inovação na indústria farmacêutica: uma janela de oportunidade no âmbito do complexo industrial da saúde. In: BUSS, P. M; CARVALHEIRO, J. R.; CASAS, C. P. R (Org.). Medicamentos no Brasil: inovação e acesso. Rio de Janeiro: Editora Fiocruz, 2008. p. 41-60.

GADELHA, C. A. G et al. (Coord.). Perspectivas do investimento em saúde. Rio de Janeiro: UFRJ, Instituto de Economia, 2008/2009. 217 p. Relatório integrante da pesquisa "Perspectivas do Investimento no Brasil", em parceria com o Instituto de Economia da UNICAMP, financiada pelo BNDES. Disponivel em: http://www.projetopib.org/?p=documentos. Acesso em 10 out. 2009.

GADELHA, C. A. G; QUENTAL, C; FIALHO, B. C. Saúde e inovação: uma abordagem sistêmica das indústrias da saúde. Cad Saúde Pública, v. 19, n. 1,p. 47-59, 2003.

GAITAN, F. 0 Desenvolvimento esquivo e as tensões do desenvolvimento. IN BOSCHI, R.R. Variedades de capitalismo, Política e Desenvolvimento na América Latina. Belo Horizonte: Editora UFMG, 2011.

HO, Paulo L. et al. Economical Value of Vaccines for the Developing Countries-The Case of Instituto Butantan, a Public Institution in Brazil. PLoS. Negl. Trop. Dis., v. 5, n. 11, e1300, 2011. doi:10.1371/ journal.pntd.0001300. 
HOCHMAN, Gilberto. A era do saneamento: as bases da política de saúde pública no Brasil. São Paulo: Hucitec; Anpocs, 1998.

IBAÑEZ, N.; WEN, F. H.; FERNANDES, S. C. G. A autossuficiência na produção de imunobiológicos e a criação do Centro de Biotecnologia do Instituto Butantan. Cadernos de História da Ciência, v. 3, n. 1, jan./jun. 2007.

IBANEZ, Ni HUI, F.W. FERNANDES, S.C.G. Instituto Butantan: história institucional (Desenho metodológico para uma periodização preliminar).Cadernos de História da Ciência,o v.1, n. 1, jan. / junh. 2005.

MALLO, Eduardo. Políticas de ciencia y tecnologia en la Argentina: la diversificación de problemas globales, ¿soluciones locales? EM: REDES. Revista de estudios sociales de la ciencia. v. 17, n. 32, p. 133-160, Buenos Aires, jun. 2011..

MARQUES, M. B. Ciência, tecnologia, saúde e desenvolvimento sustentado. Rio de Janeiro: FIOCRUZ, 1991

MOTOYAMA, S. (Org.). Prelúdio para uma história. Ciência e Tecnologia no Brasil. São Paulo: EDUSP; FAPESP,2004.

MOTOYAMA, S. FAPESP Uma História de Política Científica e Tecnológica. São Paulo:EDUSP Fapesp, 1999.

OLIVEIRA, J. L. Cronologia do Instituto Butantan (188819810); $1^{\text {a }}$ parte $1888-1.945$. .Memórias do Instituto Butantan., p 44-45, 1980-1981. p.

PLONSKI, G. A. Bases para um movimento pela inovação tecnológica no Brasil. São Paulo em perspectiva, v. 19, n.1, p. 25-33, jan./mar. 2005.

RAW, I. eDécada de 80: 0 programa de auto suficiência em imunobiológicos e o SUS. [entrevista cedida a] Laboratório Especial de História da Ciência do Instituto Butantan. Cadernos de História da Ciência, v. 3, n. 1, jan./jun.2007

RAW, I. Relatório e Perspectivas, 2003 - 2004, Instituto Butantan/Fundação Butantan. Cópia impressa, 2003.

RAW, I.; HIGASHI, H. G.; MERCADANTE, O.A. P\&D em vacinas e soros no âmbito do Instituto Butantan. Instituto/Fundação Butantan. São Paulo, 2004 
TEIXEIRA, L. A. Repensando a História do Instituto Butantan. In: DANTES, Maria Amélia M. (Org.).Espaços da Ciência no Brasil: 1800-1930..Rio de Janeiro: Editora Fiocruz, 2001.

TEMPORÃO, J.G. A propaganda de medicamentos e o mito da saúde. Rio de Janeiro: Graal, 1986.

VIANA, A. L. d'Á. et al. Saúde, desenvolvimento e inovação tecnológica: nova perspectiva de abordagem e de investigação. Lua nova, n. 83, p. 41-77, 2011.

VIANA, A. L. d'Á.; NUNES, A. A.; SILVA, H. P. Complexo produtivo da saúde, desenvolvimento e incorporação de tecnologias. In: IBAÑEZ, N.; ELIAS, P. E. M.; SEIXAS, P. H. D'Â. (Org.) Política e gestão pública em saúde. São Paulo: Hucitec; Editora: Cealag, 2011.

VIANA, A. L. D et al. Inovação e Saúde: Capacidade regulatória público-privada em dois processos tecnológicos: 1. Organizações Sociais de Saúde (OSS); 2. Tomografia Computadorizada por Emissão de Pósitrons (PET-CT): Relatório Final de Pesquisa Convênio FAPESP-CNPq-SUS (PP-SUS 2006/2007). São Paulo, 2009, 271p.

VIANA, A. L. d'Á; BOUSOUAT, A.; IBAÑEZ, N. (orgs.). Saúde, desenvolvimento, ciência, tecnologia e inovação. São Paulo: Hucitec ; Cealag, 2012

WEN, F.H. 0 Hospital Vital Brazil e o Programa de Controle de Acidentes por Animais Peçonhentos. In:Encontro Nacional dos Laboratórios Produtores de Soros e do Programa de Controle de Acidentes por Animais Peçonhentos. São Paulo: Instituto Butantan, 2003. 